\title{
The effect of background familiarity in visual search: An analysis of underlying factors
}

\author{
JOHN T. RICHARDS and GERALD M. REICHER \\ University of Oregon, Eugene, Oregon 97403
}

\begin{abstract}
The speed and accuracy of visual target search are strongly dependent on the familiarity of the background through which the search proceeds. Search through unfamiliar elements is much more difficult than search through familiar ones. This effect of background familiarity is examined in a series of three experiments. Experiment 1 suggests that the effect is not attributable to an inherently slow classification of individually unfamiliar nontargets. Experiments 2 and 3 investigate three aspects of multicharacter processing potentially sensitive to background familiarity. The results suggest that the background familiarity effect is most parsimoniously viewed as the result of slow or inaccurate segmentation of features extracted from adjacent characters. Mechanisms linking the familiarity of the background with the efficiency of character segregation are discussed in closing.
\end{abstract}

In an earlier report, Reicher, Snyder, and Richards (1976) demonstrated that the speed and accuracy of visual target search are strongly dependent on the familiarity of the background through which the search proceeds. It is, for example, much easier to search for an unfamiliar character in a matrix of familiar ones than to search for a familiar character among unfamiliar ones (cf. Frith, 1974). This effect of familiarity on visual search performance is substantial (search through familiar background elements being as much as $90 \%$ faster) and quite readily obtained; it matters little whether familiarity is varied by way of letter inversion, letter element deletion, or the use of novel character sets. Moreover, the effect is relatively independent of the familiarity of the target.

Our interest in this background-familiarity effect derives in part from its stability. In the experiments conducted by Reicher et al., unfamiliar matrices were comprised of characters drawn from a set of (at most) 12 items. Over the course of the experimental session, these unfamiliar characters were seen many times. Nevertheless, the difference between familiar and unfamiliar backgrounds did not systematically diminish over block of testing (a feature equally characteristic of the data to be presented here). This suggests that the requisite familiarity difference is either considerably larger or of a somewhat different sort than that which is easily altered within the experimental time frame. "Familiar"' characters, in other words, must be exceedingly well learned.

This research was conducted at the University of Oregon with the support of the Advanced Research Projects Agency of the Department of Defense and was monitored by the U.S. Air Force Office of Scientific Research under Contract F44620-73-C-0056. The authors thank Tom Carr, Barbara Dosher, John Duncan, and Mike Posner for helpful comments on an earlier draft.
Our interest in the effect of background familiarity has been further motivated by the nonexistence of single-character familiarity effects-effects from which the multicharacter matrix results could be derived by extrapolation. In Reicher et al.'s sixth experiment, single characters were classified on each trial as either "target" (an uppercase K) or "nontarget." In separate blocks, either digits (familiar stimuli) or Gibson figures (unfamiliar stimuli) served as nontargets. Classification was slightly (but not significantly) faster for single unfamiliar nontargets. Even so, a subsequent matrix-search experiment confirmed that search for an uppercase $\mathrm{K}$ amidst Gibson figures was over $60 \%$ slower than search through digits (Reicher et al., 1976, Experiment 7). This suggests that the effect of background familiarity arises through some means other than the concatenation of differences in isolated-character performance.

A replication of this null result with single characters was considered prerequisite to the continued investigation of background familiarity. Thus, in Experiment 1, a single-character classification task was embedded within the context of a standard matrix search, one in which the number of matrix elements was parametrically varied from one to many. Using these data, we may examine the extent to which slow search through unfamiliar matrices can be attributed to an inherently slow classification of unfamiliar nontargets.

\section{EXPERIMENT 1}

\section{Method}

Subjects. Eight volunteers were recruited from a subject pool at the University of Oregon. All possessed normal or corrected vision and were native speakers of American English. While most had participated in other studies of visual information acquisition, 
none had previously participated in search tasks similar to the current one. All were paid $\$ 2$ for each of two 50-min sessions.

Apparatus. All predisplay warning signals, test displays, and postresponse feedback were presented on a cathode-ray tube under computer control. All stimuli were well above threshold. Subjects viewed this display from a distance of approximately $45 \mathrm{~cm}$. Response choice on each trial was registered by pressing one of two keys. This apparatus was used throughout the three experiments reported here.

Procedure. Following a brief explanation of the experimental task and apparatus, each subject completed a series of 576 trials (six blocks of 96 trials) on each of 2 consecutive days. On the Ist day of testing, a randomly selected half of the subjects searched for any of a set of unfamiliar targets embedded in matrices of familiar elements. The remaining subjects searched for any of a set of familiar targets in matrices of unfamiliar elements. On the 2nd day of testing, this assignment was reversed. Rest opportunities were provided every 32 trials by an interruption of the betweentrial sequencing. Subjects were encouraged to rest following each 96-trial block.

Familiar elements consisted of the set of 12 uppercase letters: A, B, C, E, F, G, K, L, P, R, T, and Y, each letter configured from light points arrayed within a five $X$ seven rectangular dotmatrix. Letters subtended approximately $.24^{\circ}$ vertically and $.16^{\circ}$ horizontally. Unfamiliar elements consisted of the same set of letters rotated $180^{\circ}$ in the plane of the display face.

Stimuli consisted of $1,3,6$, or 12 letters, the number of letters varying pseudorandomly over trials (see Figure 1). Each matrix size occurred 24 times within each block of 96 trials. Letters were arrayed within a 12-position circular matrix (clockface), each letter's midpoint separated from the fixation point by a visual angle of approximately $.80^{\circ}$. Single characters (i.e., arrays of size 1) were plotted equally often in each of the 12 matrix positions. Arrays of size 3 were plotted equally often in the four ways in which three equally spaced items can be positioned. Arrays of size 6 were plotted equally often in the two ways in which six equally spaced items can be positioned. Arrays of size 12 filled the circular matrix. Targets-presented on exactly half the trialsappeared equally often at each matrix position within each array size. Each block of 96 trials thus represented one complete replication of all crossed factors save background type.

Prior to each trial, characters were randomly assigned to the appropriate matrix positions. In the familiar-background (upright letter) condition, targets were randomly drawn from the set of inverted letters. In the unfamiliar-background (inverted letter) condition, targets were drawn from the set of upright letters. For any given trial, either one target or none was assigned to the stimulus array. Background elements were selected without replacement from the appropriate character set. In this and all subsequent experiments, no letter appeared in any given array as both an upright and rotated character.

Each trial began with the presentation of a central fixation

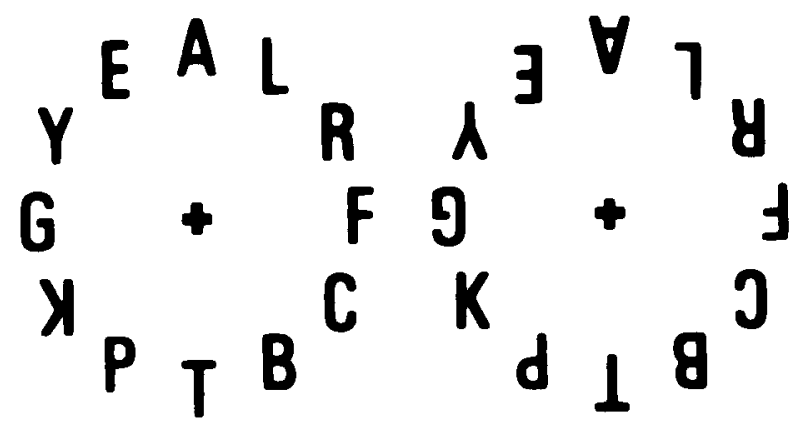

Figure 1. Familiar and unfamiliar 12-item matrices as used in Experiment 1 (approximately to scale). Note the targets at eight o'clock.

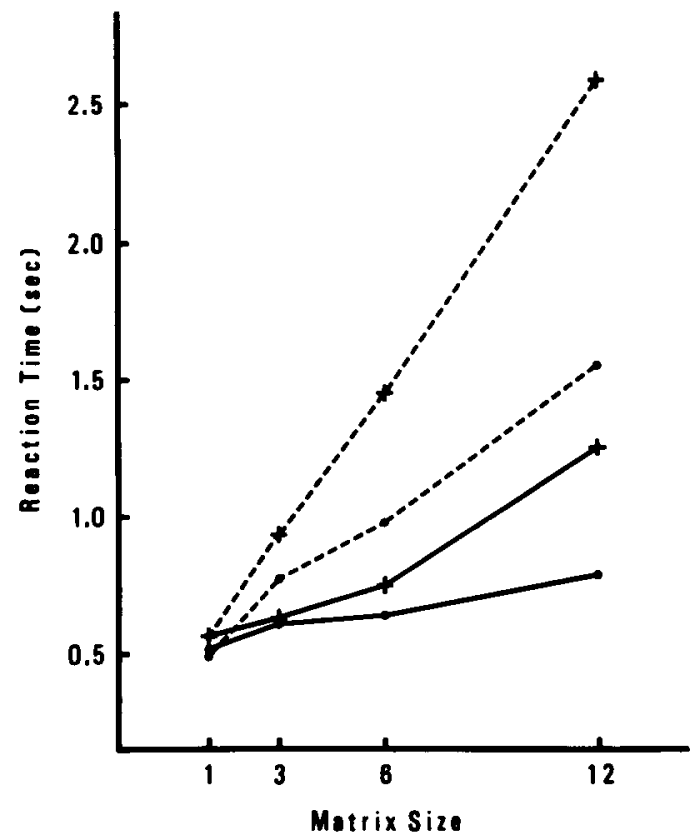

Figure 2. Median RT from Experiment 1 as a function of number of matrix elements. Solid lines represent upright backgrounds. Dashed lines represent rotated backgrounds. Data from target-present trials are plotted with disks. Data from targetabsent trials are plotted with crosses. (Note that matrices of size 1 consisted of a single target on target-present trials and a single background character on target-absent trials.)

marker (+) for $500 \mathrm{msec}$. The stimulus array was plotted $500 \mathrm{msec}$ after the offset of this marker. Array elements remained on the display screen until a keypress indicating the presumed presence (Yes) or absence (No) of a target was made (note that a target was any member of the 12-item target set). Subjects pressed the Yes key with the preferred finger of one hand and the No key with the other hand, but no attempt was made to counterbalance hand assignment over hand of preference (this need not concern us, since only differences between conditions will be compared across response type). Immediately following each response, reaction time (RT) and error feedback were displayed for $500 \mathrm{msec}$. A new trial began $1.5 \mathrm{sec}$ later.

\section{Results and Discussion}

Given the evidence cited above (Reicher et al., 1976, Experiments 6 and 7), we hypothesized that the estimated effects of nontarget familiarity (derived for single nontargets from the multi-letter array data) would be significantly larger than the effect actually obtained when only single nontargets were presented for classification.

Search time is summarized in Figure 2. The effect of nontarget familiarity may be estimated from these data by dividing the magnitude of each multicharacter familiarity effect by the appropriate number of array elements (i.e., by subtracting the average response time for a familiar background of size $\mathrm{n}$ from the response time for the corresponding unfamiliar background, and dividing this quantity by $n$ ). Note that these estimates need not be corrected for the presence of a target character; since familiar targets were 
classified slightly faster than unfamiliar targets (492 msec with $3.5 \%$ error vs. 520 msec with $9.2 \%$ error, the error difference significant by sign test with $\mathrm{p} \simeq$.016), and since familiar targets were embedded in unfamiliar surrounds, our estimates of the effect of nontarget familiarity are conservative.

By this procedure, single-character familiarity effects in the target-present data were estimated to be $54 \mathrm{msec}$ for arrays of size 3,57 $\mathrm{msec}$ for arrays of size 6 , and $64 \mathrm{msec}$ for arrays of size 12 . In the target-absent data, these same effects were estimated to be 103, 117, and $111 \mathrm{msec}$. Even the smallest of these is significantly larger than the effect actually obtained when only a single nontarget was presented for classification [the null hypothesis may be rejected by a one sample $t$ test, with $t(7)=2.00, p<.05$, one-tailed]. Indeed, the actual difference in single nontarget classification time was slightly negative (561 msec with 5.9\% error for unfamiliar nontargets vs. $564 \mathrm{msec}$ with $9.0 \%$ error for familiar nontargets, the difference in error rate significant by sign test with $\mathrm{p}=.035$ ). Thus we may reject the hypothesis that the effects of background familiarity reflect the mere concatenation of single-character familiarity effects.

Overall performance is summarized in Figure 2 and Table 1. Analyses of variance ${ }^{1}$ confirmed that unfamiliar backgrounds were searched more slowly than familiar backgrounds $[F(1,6)=28.25, \mathrm{p}<.005$, in the target-present data; $F(1,6)=68.80, p<.001$, in the target-absent data], that search time increased with increasing array size $[F(3,18)=48.46, p<.001$, in the target-present data; $F(3,18)=67.93, p<.001$, in the target-absent data], and that this increase was more pronounced for unfamiliar matrices $[\mathrm{F}(3,18)$ $=33.53, \mathrm{p}<.001$, in the target-present data; $F(3,18)$ $=44.45, \mathrm{p}<.001$, in the target-absent data]. Search accuracy proved somewhat more variable. Although targets were detected in familiar backgrounds with slightly greater accuracy than in unfamiliar backgrounds, this difference was not significant. Moreover, since the target-present response was incorrectly produced (i.e., false alarms were made) more frequently to matrices comprised of familiar elements, $\mathrm{F}(1,6)=16.04, \mathrm{p}<.01$, this slight detection advantage was probably due to response bias. In agreement with the RT data, however, targets were detected more frequently in matrices containing fewer elements, $F(3,18)=8.38, p<.005$, and this effect of matrix size was greater for unfamiliar backgrounds, $F(3,18)=6.98, p<.005$.

In summary, the first experinient has confirmed that slow search through unfamiliar backgrounds is not attributable to an inherently slow classification of unfamiliar nontargets. In an otherwise empty field, unfamiliar nontargets are classified as rapidly as familiar ones. We may thus be reasonably certain
Table 1

Percent Error in Experiment 1 as a Function of Background Familiarity, Matrix Size, and Target Presence

\begin{tabular}{|c|c|c|c|c|c|}
\hline \multirow[b]{2}{*}{ Trial Type } & \multicolumn{4}{|c|}{$\begin{array}{c}\text { Matrix Size } \\
\text { (in number of characters) }\end{array}$} & \multirow[b]{2}{*}{ Mear } \\
\hline & $1^{*}$ & 3 & 6 & 12 & \\
\hline \multicolumn{6}{|c|}{ Target Present } \\
\hline Familiar & 9.2 & 8.3 & 9.7 & 17.2 & 11.1 \\
\hline Unfamiliar & 3.5 & 11.8 & 12.8 & 21.8 & 12.5 \\
\hline \multicolumn{6}{|l|}{ Target Absent } \\
\hline Familiar & 9.0 & 8.1 & 4.3 & 7.6 & 7.3 \\
\hline Unfamiliar & 5.9 & 2.9 & 3.5 & 5.5 & 4.5 \\
\hline
\end{tabular}

*Matrices of Size 1 consisted of a single target on target-present trials and a single background character on target-absent trials.

that some aspects of multicharacter processing gives rise to the background-familiarity effect.

Of all the problems uniquely tied to the processing of multicharacter fields, at least three are plausibly affected by the familiarity of the background. First, multiple characters must be individually resolved. That is, features must be appropriately grouped (by character) and groups must be clearly delineated. If unfamiliar characters are less readily parsed into feature groups, or if the resulting groups are more subject to an interchange of features over time, the efficiency of search could suffer. Second, multiple characters must share a common pool of representational resources. If this pool is limited, an increase in the resources utilized by one character will lead to a decrease in the resources concurrently available to other characters. Any such change will be reflected in slower search, lower accuracy, or some combination of both. The effect of background familiarity could be explained within this framework by assuming that unfamiliar characters require more substantial representational support than familiar ones. Finally, multiple characters (if they cannot be examined simultaneously) must be examined in an order which maximizes the probability of target detection. For the matrices used in our experiments, the sequential classification of adjacent characters represents the optimal search path. Any deviation from this path will necessarily lessen the efficiency of search. If the order of examination is tied to the trajectory of visual attention, and if visual attention is summoned by novel or unfamiliar stimuli, the backgroundfamiliarity effect could result. The next two experiments will examine these interpretations.

\section{EXPERIMENT 2}

In this second experiment, single characters were presented in rapid succession. Subjects monitored the resulting character streams (which consisted of either familiar or unfamiliar nontargets) for the presence of target stimuli. If the effect of background familiarity 
(in spatially extended arrays) results from the summoning of visual attention to locations containing unfamiliar nontargets, then no effect of stream familiarity is to be expected. This follows quite simply from the fact that the order of character examination is no longer under subject control. Any effect of stream familiarity may thus be attributed to one of the remaining two mechanisms. ${ }^{2}$

\section{Method}

Subjects. Eight right-handed volunteers were recruited as before. Each was paid for participation in one 50-min experimental session.

Procedure. Following a period of task orientation, the subjects underwent a series of $\mathbf{5 7 6}$ trials. During the first three blocks of 96 trials, half the subjects monitored a sequential stream of familiar characters for the presence of any of a set of unfamiliar characters. The remaining subjects monitored a stream of unfamiliar characters for the presence of any of a set of familiar characters. During the last three blocks, this assignment was reversed. The subjects were reminded of target type (upright or rotated letter) prior to each block of testing. Rest opportunities were provided every 32 trials.

Familiar and unfamiliar characters were drawn from the uprightand rotated-letter sets of Experiment 1. Single letters-each subtending $.24^{\circ}$ vertically and $.16^{\circ}$ horizontally-were sequentially generated in the same central display position. Within each display sequence, letters were presented every $125 \mathrm{msec}$.

Procedurally, this experiment may be usefully conceptualized as a 12-item matrix extended in time rather than space. Each trial began, as before, with the presentation of a 500 -msec warning signal followed $500 \mathrm{msec}$ later by a sequence of 12 letters. Letter presentation required $1.5 \mathrm{sec}$ ( $125 \mathrm{msec}$ times 12 letters). On $75 \%$ of all trials, one of these letters was a member of the target set. Targets were presented equally often in each of the 12 temporal positions. Selection of target and background characters proceeded exactly as specified for the 12-item matrices of Experiment 1.

During each display sequence, the subjects pressed a response key with the right hand as soon as a target letter was detected. RT was measured from the onset of the target stimulus to the registration of the keypress. RTs of less than $150 \mathrm{msec}$ were classified as anticipations and were not included in subsequent RT analysis. If, at the end of the sequence, no target had been detected, the subjects pressed an adjacent key with the left hand. The subjects were encouraged to respond rapidly following target detection, but speed was not stressed for nontarget responses. Following each sequence, the subjects were informed of response accuracy and (on correct detection trials) response speed. Feedback was displayed for $1 \mathrm{sec}$ at the completion of letter presentation (if the target key had been depressed) or upon registration of the nontarget response. A new trial began $1 \mathrm{sec}$ later.

\section{Results and Discussion}

Three dependent measures from the target-present trials (proportion targets missed, median RT, and anticipation rate) and one measure from the targetabsent trials (false-alarm rate) were subjected to separate analyses of variance. ${ }^{3}$

Proportion targets missed. A large and significant effect of background familiarity was obtained in the miss data. Of the targets embedded in familiar streams, $10.2 \%$ were not detected. Of those in unfamiliar streams, $21.1 \%$ were missed, $F(1,6)=$ $15.93, \mathrm{p}<.01$.

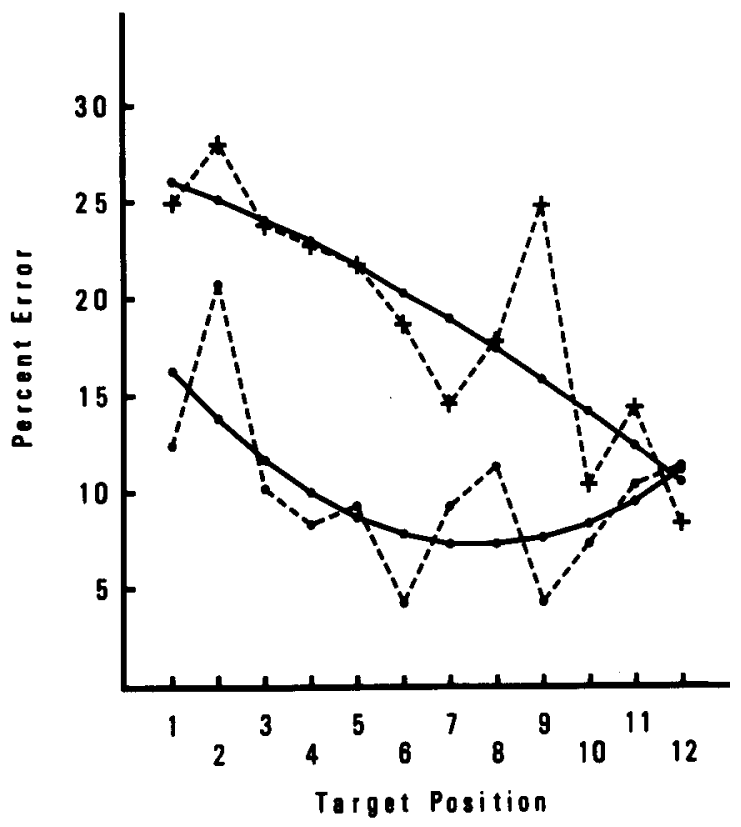

Figure 3. Percent targets missed in Experiment 2 as a function of temporal target position (each percentage based on 144 trials). Upright background streams yielded the error rates plotted with disks. Rotated background streams yielded the rates plotted with crosses. The convergence of these two functions is most readily seen in the second-order polynomials (solid lines) fit to the means.

Post hoc analysis of the temporal target-position data revealed the pattern shown in Figure 3. Targets presented in the first nine temporal positions were detected with greater frequency when embedded among familiar letters (the pairwise differences significant by Scheffé test for all but the eighth position). Interestingly, this advantage was lessened for targets presented in Positions 10 and 11 and reversed for targets in Position 12 (the magnitude of the reversal falling just short of significance). The reliability of the overall diminution was indicated by a significant interaction of background familiarity with the linear component of temporal position, $F(1,6)=9.63$, $\mathrm{p}<.025$ [background familiarity also interacted with the quadratic component of temporal position; $F(1,6)=8.42, p<.05]$. The convergence of these two functions suggests that the detection decrement observed in unfamiliar background streams depends in some way on the read-in of at least one unfamiliar nontarget subsequent to target presentation.

Median RT. Background familiarity had little effect on the speed of detection report. Median report time in familiar $(596 \mathrm{msec})$ and unfamiliar (609 msec) backgrounds did not significantly differ, $F(1,6)<1$, although an effect of background order was observed; subjects receiving upright backgrounds during the first three blocks of testing responded more rapidly (across all other factors, including background type) than subjects initially 
receiving rotated backgrounds [530 vs. $675 \mathrm{msec}$, $F(1,6)=8.78, p<.05]$. The absence of RT effects is not particularly troubling. Rigid stepping of character input might well force a constant time course on the processes of target detection (all effects being evidenced, therefore, in differential detection accuracy). Were input rate under subject control, one might reasonably expect a trading off of speed against accuracy, search slowing to raise accuracy to acceptable levels.

Anticipation rate. There was no differential tendency to anticipate target onset as a function of background type. Anticipations represented $3.7 \%$ of the familiar-background data and $4.0 \%$ of the unfamiliarbackground data, $F(1,6)<1$.

False-alarm rate. Background differences were not evidenced in the target-absent data. Subjects responded incorrectly on $22.6 \%$ of the familiarbackground and $23.1 \%$ of the unfamiliar-background trials, $F(1,6)<1$. Although the overall false-alarm rate is rather high (due, perhaps, to the speeded nature of the detection report and the high probability of target presentation), it is clear that these data will not compromise our interpretation of the background effect in the miss data.

The results of Experiment 2 suggest that the effect of background familiarity is not due to the nonsequential examination of unfamiliar matrices. While nonsequential search may contribute to the familiarity effect in spatially extended arrays, it cannot account for the effect of stream familiarity in the present data.

\section{EXPERIMENT 3}

Two interpretations of the background-familiarity effect remain. Either can account for the results of Experiments 1 and 2. Let us review them in turn.

The first interpretation places the effect of background familiarity during the early partitioning of features into character groups. Note that partitioning need not occur when single characters are presented in isolation. Thus, no effect of single-character familiarity is predicted by this model. The effect of background familiarity in spatially extended matrices is interpreted as the result of slow or inaccurate segmentation of the features extracted from adjacent characters. The effect of stream familiarity in temporally extended arrays is viewed as the result of confusions between successive characters. It is suggested that both forms of cross-group feature assignment are more probable (or that the correct assignment of features to potentially confused groups takes more time) when the features are extracted from unfamiliar characters.

The second interpretation places the effect of background familiarity in the sharing of a limited pool of representational resources. For our present purposes, the form of support provided by this pool need not be specified. Only the amount of support available need be explicitly assumed. To account for the absence of single-character effects, the pool must be able to support the processing of one unfamiliar character. Three characters must begin to overtax it (see Figure 2). If these limits are maintained, and if we assume that unfamiliar characters demand more substantial support than familiar ones, the results of Experiments 1 and 2 follow.

Experiment 3 was designed to contrast these two interpretations. Targets were embedded in spatially extended matrices configured to permit the independent variation of array density (manipulated by intercharacter distance) and the number of target-flanking background elements. The number of characters within each matrix remained constant at three. If the effect of background familiarity arises during character segregation, we may expect the effect to interact with both the density of character packing and the number of target-flanking elements. The diminution of target detectability produced by placing background elements nearer to the target (see, e.g., Eriksen \& Eriksen, 1974) should be more pronounced when the elements are unfamiliar. Similarly, the diminution produced by placing the target between two background elements (see, e.g., Townsend, Taylor, \& Brown, 1971) should be more pronounced in unfamiliar arrays. These interactions are not to be expected if the background-familiarity effect results from differential resource demand.

\section{Method}

Subjects. Twelve right-handed volunteers were selected in the usual manner.

Procedure. Following task familiarization, subjects received eight blocks of 72 trials. Blocks 1 and 5 were treated as practice and were not included in subsequent analyses. Background familiarity was held constant over the first four and last four blocks of testing. Background order (upright blocks preceding rotated blocks vs. rotated preceding upright) and hand assignment (right vs. left key for the target-present response) were factorially crossed over subjects. Rest breaks were provided every 24 trials.

Familiar and unfamiliar characters were drawn randomly and without replacement (prior to each trial) from the upright- and rotated-letter sets used previously. Characters subtended approximately $.24^{\circ}$ vertically and $.16^{\circ}$ horizontally. Character triples were arrayed about an imaginary clockface, each character positioned $.80^{\circ}$ from fixation. Array items occupied adjacent positions on a randomly selected half of the trials. Within these highdensity matrices, characters were presented equally often in each of the following configurations: 10,11 , and 12 (o'clock); 11,12 , and $1 ; 12,1$, and $2 ; 8,7$, and $6 ; 7,6$, and $5 ; 6,5$, and 4 . Characters were separated on remaining trials by one empty clock position. In these low-density matrices, characters were presented equally often in these configurations: 9,11 , and $1 ; 10,12$, and 2 ; 11,1 , and $3 ; 9,7$, and $5 ; 8,6$, and $4 ; 7,5$, and 3 . Targets (upright letters in rotated backgrounds, rotated letters in upright backgrounds) were presented on half the trials. Targets were positioned equally often in each of the three character positions within each of the 12 matrix configurations.

A fixation character $(+)$ of $500 \mathrm{msec}$ duration preceded matrix 
onset by $1 \mathrm{sec}$. Matrices remained at full display intensity for $50 \mathrm{msec}$, decaying to $.1 \%$ of this intensity in an additional $32 \mathrm{msec}$. Subjects indicated the presumed presence or absence of a target by pressing either of two keys (counterbalanced as outlined above) and were encouraged to respond as quickly and accurately as possible. Following each response, RT and error feedback were displayed for $500 \mathrm{msec}$. The next trial began $1.5 \mathrm{sec}$ later.

\section{Results}

Aside from a reduction in overall RT with practice $[F(2,20)=4.55, p<.025$, in the target-present data $]$, all differences in target detectability were confined to the error data. Nevertheless (as shown in Figures 4 and 5), median RT and percent error rate traced generally parallel functions.

Analysis of the miss data ${ }^{4}$ indicated that background familiarity interacted (as predicted by the character-segregation model) with both array density, $\mathrm{F}(1,10)=5.77, \mathrm{p}<.05$, and target position, $\mathrm{F}(2,20)$ $=4.77, p<.025$. No other effects reached significance.

Figure 4 depicts detection performance as a function of background familiarity and array density. Examination of the individual error data confirmed that the decrease in target detectability (with increasing array density) was greater for targets embedded in unfamiliar arrays. This pattern was evident in the error data of 9 of 11 subjects (with one tie, $p \simeq .033$ by sign test). Interpretation of this interaction is somewhat complicated by the presence of a Background by Density interaction in the target-absent data. Target-present responses were incorrectly produced on $33.6 \%$ of the upright-background/highdensity trials, $30.4 \%$ of the upright/low-density trials, $24.2 \%$ of the rotated/high-density trials, and $27.1 \%$

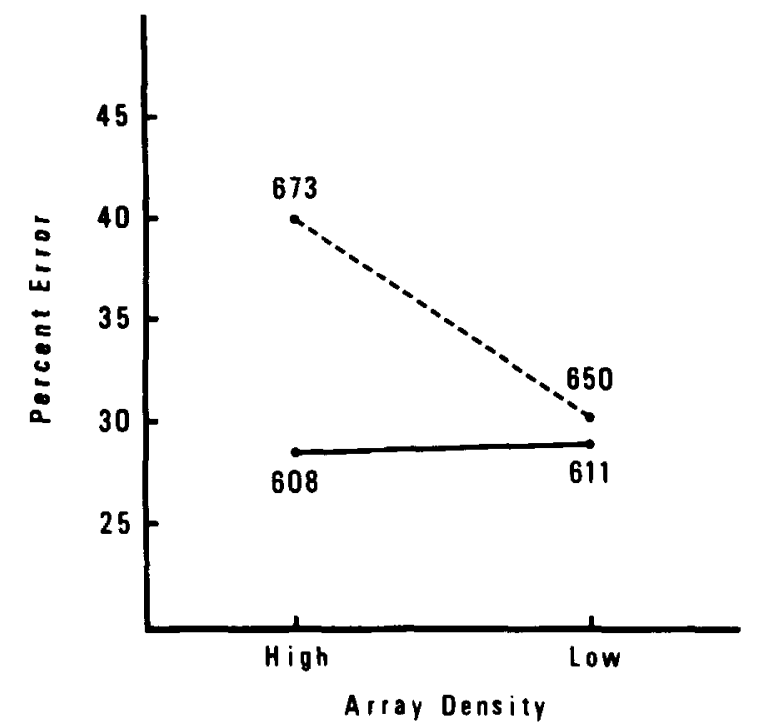

Figure 4. Percent targets missed and median RT (from Experiment 3) as functions of array density. Data are plotted separately for upright (solid line) and rotated (dashed line) backgrounds.

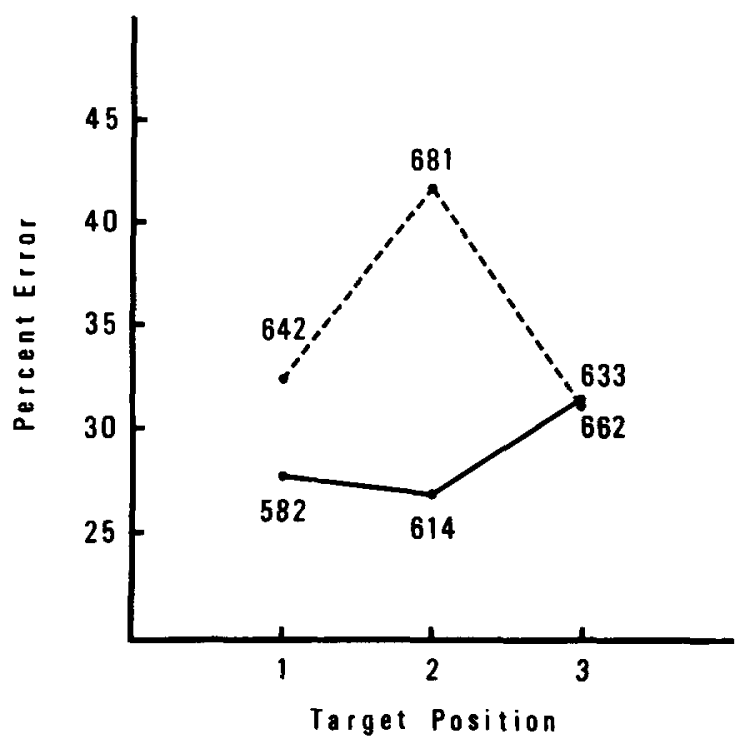

Figure 5. Percent targets missed and median RT (from Experiment 3) as functions of target position. Data are plotted for upright (solid line) and rotated (dashed line) backgrounds. (The 662-msec response time at Target Position 3 is associated with the rotated-background data.

of the rotated/low-density trials, $F(1,10)=7.67$, $\mathrm{p}<.025$. All such differences in false-alarm rate were confined, however, to subjects receiving upright stimuli in the first four blocks of testing, $F(1,10)=$ $13.04, \mathrm{p}<.005$. More importantly, those subjects exhibiting the smallest differences in the target-absent data were those showing the largest interactive effects of background familiarity and array density in the target-present data $\left(r_{s}=-.59, p \simeq .024\right)$. Thus the interaction depicted in Figure 4 cannot be attributed to differences in false-alarm rate.

Figure 5 summarizes the interactive effects of background familiarity and target position (note that position numbering proceeds from leftmost to rightmost character, target position being averaged in this way to pick up any residual left-to-right processing bias). Examination of the individual error data revealed greater effects of dual target-flanking when the surrounding elements were unfamiliar; the difference (in targets missed) between Target Position 2 and the average of Target Positions 1 and 3 was larger in the unfamiliar background data of 10 of 11 subjects (with one tie, $p \simeq .006$ by sign test).

\section{DISCUSSION}

The spatial interactions of Experiment 3 are not predicted by the limited-resource interpretation sketched above. While unfamiliar characters may demand a greater share of resources under some conditions, these data argue that such a mechanism can account only partially for the overall effect of background familiarity. It is suggested, therefore, 
that slow or inaccurate character segregation contributes to the slowing of search in unfamiliar matrices. In this final section we will consider how the efficiency of segregation might be tied to the familiarity of the background field.

\section{Feature Perturbation}

Wolford (1975) has suggested that interletter interference during tachistoscopic recognition may result from perturbations in the grouping of spatially bundled features. His model assumes that features are first extracted (in parallel via independent channels) and then parsed into groups (groups being delimited by "space" features). Features thus ordered are subject to perturbation across group boundaries, the probability of perturbation increasing with distance from fovea, density of array, and time since extraction.

The results of Experiment 3 may be handled within this framework by assuming the existence of various strengths of interfeature binding. In this view, features would be linked not merely by spatial contiguity but by the degree to which they were associated. Highly familiar stimuli would tend to resist the loss of features. Unfamiliar stimuli (being bound by common location only) would manifest less resistance to outward perturbation. Since the number of freefloating features would be greater for fields of unfamiliar stimuli, we should expect the effect of array density to be larger for rotated-letter surrounds. The effect of dual target-flanking would be similarly accentuated. This could account for the results of Experiment 3, and by extension, the general effects of background familiarity.

\section{Level of Representation}

The process of character familiarization may be conceptualized as a progressive strengthening of interfeature links. Alternatively, the process may involve the creation of high-level representations (not necessarily verbal) which come to stand for structured conjunctions of lower-level features. Once established, these compact codes could be accessed and efficiently manipulated as singular entities (cf. LaBerge, 1973; Treisman, Sykes, \& Gelade, 1977).

Prior to the formation of conjunction codes, unfamiliar stimuli must be represented as multifeature composites-bundles, essentially, of primitive character elements and their interrelations. Projected on a common surface, such bundles might yield a cognitive matrix of greater effective density. Note carefully that each character in the distal matrix might be fully represented by its corresponding feature group. We need not posit a loss of character information to account for the reduction in search efficiency associated with unfamiliar matrices. We need only assume that adjacent character representations are less readily segregated when they are crowded in either the distal or cognitive matrices. Given this assumption, the interactions of Experiment 3 follow.

\section{REFERENCES}

ERIKSEN, B. A., \& Erirsen, C. W. Effects of noise letters upon the identification of a target letter in a nonsearch task. Perception \& Psychophysics, 1974, 16, 143-149.

FRrTt, U. A curious effect with reversed letters explained by a theory of schema. Perception \& Psychophysics, 1974, 16, 113-116.

LABerge, D. Attention and the measurement of perceptual learning. Memory \& Cognition, 1973, 1, 268-276.

Reicher, G. M., Swyder, C. R. R., \& Richards, J. T. Familiarity of background characters in visual scanning. Journal of Experimental Psychology: Human Penception and Performance, 1976, 2, 522-530.

Townsend, J. T., TAYLOR, S. G., \& BRown, D. R. Lateral masking for letters with unlimited viewing time. Perception \& Psychophysics, 1971, 10, 375-378.

Treisman, A. M., Sykes, M., \& Grlade, G. Selective attention and stimulus integration. In S. Dornic (Ed.), Attention and performance VI. Potomac, Md: Erlbaum, 1977.

Wolford, G. Perturbation model for letter identification. Psychological Review, 1975, 82, 184-199.

\section{NOTES}

1. The RT and error data from the target-present and targetabsent trials were separately analyzed using a mixed design with one between-subjects factor (order of background type) and three within-subjects factors (background type, array size, and block of testing). In this and all subsequent analyses, proportions were entered following arc sine transformation.

2. The RT data of Experiment 1 suggest that matrix search followed a serial and self-terminating course (observe that the slopes of the target-absent functions are roughly twice those of the targetpresent functions - a fact reflected in the magnitudes of the estimated single-character familiarity effects). Serial, self-terminating search is not consistent with the hypothesized direction of attention to locations containing unfamiliar stimuli. Corroborating evidence was nevertheless desired.

3. All four dependent measures were separately analyzed using a mixed design with one between-subjects factor (order of background type) and two within-subjects factors (background type and block of testing). The analysis of temporal target-position utilized a mixed design with one between-subjects factor (order of background type) and two within-subjects factors (background type and temporal target-position), temporal position being further partitioned into its first three (orthogonal) polynomial components.

4. Target-present data were analyzed using a mixed design with one between-subjects factor (order of background type) and four within-subjects factors (background type, array density, target position, and block of testing). Analysis of the target-absent data was based on a mixed design without the target-position factor.

(Received for publication November 28, 1977; revision accepted April 3, 1978.) 